\title{
Correction to: Cerebellar Modules and Their Role as Operational Cerebellar Processing Units: A Consensus paper
}

\author{
Richard Apps ${ }^{1}$ - Richard Hawkes ${ }^{2}$. Sho Aoki ${ }^{3,4}$ - Fredrik Bengtsson ${ }^{5}$ - Amanda M. Brown ${ }^{6,7,8}$. Gang Chen ${ }^{9}$. \\ Timothy J. Ebner ${ }^{9} \cdot$ Philippe Isope $^{10}$. Henrik Jörntell ${ }^{5}$. Elizabeth P. Lackey ${ }^{6,7,8}$. Charlotte Lawrenson ${ }^{1}$. \\ Bridget Lumb ${ }^{1} \cdot$ Martijn Schonewille ${ }^{4} \cdot$ Roy V. Sillitoe ${ }^{6,7,8,11} \cdot$ Ludovic Spaeth $^{10} \cdot$ Izumi Sugihara $^{12} \cdot$ Antoine Valera $^{10}$. \\ Jan Voogd ${ }^{4}$. Douglas R. Wylie ${ }^{13} \cdot$ Tom J. H. Ruigrok $^{4}$
}

Published online: 21 June 2018

(C) Springer Science+Business Media, LLC, part of Springer Nature 2018

\section{Correction to: Cerebellum}

https://doi.org/10.1007/s12311-018-0952-3

In the original version of this paper, the Title should have been written with "A Consensus paper".

This is hereby updated to read "Cerebellar Modules and Their Role as Operational Cerebellar Processing Units: A Consensus paper" as given above.

The online version of the original article can be found at https://doi.org/ 10.1007/s12311-018-0952-3

Tom J. H. Ruigrok

t.ruigrok@erasmusmc.nl

Richard Apps

r.apps@bristol.ac.uk

Richard Hawkes

rhawkes@ucalgary.ca

Sho Aoki

ktsky1020@yahoo.co.jp

Fredrik Bengtsson

fredrik.bengtsson@med.lu.se

Amanda M. Brown

amanda.brown@bcm.edu

Gang Chen

chenx007@umn.edu

Timothy J. Ebner

ebner001@umn.edu

Philippe Isope

philippe.isope@inci-cnrs.unistra.fr

Henrik Jörntell

henrik.jorntell@med.lu.se

Elizabeth P. Lackey

elizabeth.lackey@bcm.edu

\author{
Charlotte Lawrenson \\ pycll@bristol.ac.uk \\ Bridget Lumb \\ b.m.lumb@bristol.ac.uk \\ Martijn Schonewille \\ m.schonewille@erasmusmc.nl \\ Roy V. Sillitoe \\ sillitoe@bcm.edu \\ Ludovic Spaeth \\ 1spaeth@inci-cnrs.unistra.fr \\ Izumi Sugihara \\ isugihara.phy1@tmd.ac.jp \\ Antoine Valera \\ antoine.valera@etu.unistra.fr \\ Jan Voogd \\ janvoogd@bart.nl \\ Douglas R. Wylie \\ dwylie@ualberta.ca
}

Extended author information available on the last page of the article 


\section{Affiliations}

Richard Apps ${ }^{1} \cdot$ Richard Hawkes $^{2}$ - Sho Aoki ${ }^{3,4}$. Fredrik Bengtsson ${ }^{5}$. Amanda M. Brown ${ }^{6,7,8}$. Gang Chen ${ }^{9}$. Timothy J. Ebner ${ }^{9} \cdot$ Philippe Isope $^{10} \cdot$ Henrik Jörntell ${ }^{5}$. Elizabeth P. Lackey ${ }^{6,7,8}$. Charlotte Lawrenson ${ }^{1}$. Bridget Lumb ${ }^{1} \cdot$ Martijn Schonewille $^{4} \cdot$ Roy V. Sillitoe ${ }^{6,7,8,11} \cdot$ Ludovic Spaeth $^{10} \cdot$ Izumi Sugihara $^{12} \cdot$ Antoine Valera $^{10}$. Jan Voogd ${ }^{4} \cdot$ Douglas R. Wylie $^{13} \cdot$ Tom J. H. Ruigrok ${ }^{4}$

1 School of Physiology, Pharmacology and Neuroscience, University of Bristol, Bristol, UK

2 Hotchkiss Brain Institute, University of Calgary, Calgary, Canada

3 Neurobiology Research Unit, Okinawa Institute of Science and Technology, Onna, Japan

4 Department of Neuroscience, Erasmus MC Rotterdam, Rotterdam, the Netherlands

5 Department of Experimental Medical Sciences, Lund University, Lund, Sweden

6 Department of Pathology \& Immunology, Baylor College of Medicine, Houston, TX, USA

7 Department of Neuroscience, Baylor College of Medicine, Houston, TX, USA
8 Jan and Dan Duncan Neurological Research Institute at Texas Children's Hospital, Houston, TX, USA

9 Department of Neuroscience, University of Minnesota, Minneapolis, MN, USA

10 Institut des Neurosciences Cellulaires et Intégratives, CNRS, Université de Strasbourg, Strasbourg, France

11 Program in Developmental Biology, Baylor College of Medicine, Houston, TX, USA

12 Department of Systems Neurophysiology, Graduate School of Medical and Dental Sciences, Tokyo Medical and Dental University, Tokyo, Japan

13 Neuroscience and Mental Health Institute, University of Alberta, Edmonton, AB, Canada 